\title{
Premise Variables Cases for LMI based TS Fuzzy Observers
}

\author{
Hazerchi Mohamed ${ }^{1, *}$ and Illoul Rachid ${ }^{2}$ \\ ${ }^{1,2}$ Ecole Nationale polytechnique d'Alger, Algeria \\ ${ }^{1}$ hazerchimed@yahoo.com, ${ }^{2}$ rachid.illoul@enp.edu.dz
}

\begin{abstract}
This paper deal with the control of TS fuzzy system via fuzzy observer such as we consider the stability conditions of the augmented system ( TS fuzzy model + fuzzy observer) using different cases of the premise variables.

We propose a new classification while using the premise variables for both TS fuzzy model and fuzzy observer. We show the existing differences when using premise variable with same or different nature (either measurable or unmeasurable) for TS fuzzy model and fuzzy observer. The LMI tools are used to represent the stability conditions and the design procedure.

We present in this paper a comment about previous result of the augmented system stability (state and observer) in the unmeasurable case of the premise variable cited in [1] of Tanaka et al.
\end{abstract}

Keywords: Fuzzy TS observer; measurable; unmeasurable; premise variable

\section{Introduction}

The fuzzy TS system is very investigated and used for nonlinear systems either in control or in fuzzy observer design. In literature, we find many works which deal with TS fuzzy models and observers [1] [6] [9][11][12], usually this works deal with TS local models obtaining, the membership functions shapes, and the operating range choice.

One of the main points in fuzzy system is the premise (decision) variable choice or estimation, such as this last remind very important factor in TS fuzzy system designs because it leads the TS fuzzy models construction, because it defines the operating range and decides on the interpolation of local models, such as it defines the degree of membership function which indicates the degree of accuracy between the nonlinear system and the TS fuzzy model, in an others way the premise variable is the responsible of equivalence between the TS fuzzy system and the nonlinear system.

We distinguish two kinds of premise variables; the measurable by sensors (for example; input or output), and the unmeasurable which can be estimated through observer (for example; the states).

There are lot of works which deal with fuzzy observer stability and design, we can cite the following works:

R. J. Patton et al. [4] Proposed fuzzy Luenberger observer to be used in fault diagnosis scheme, they expressed the stability condition of fuzzy observer as eigenvalue constraint conditions.

Tanaka et al. [1] [8] proposed theorems for the stability of TS fuzzy systems with PDC (Parallel Distributed Controller) and the stabilization with PDC plus fuzzy observer, they described the design procedure in two cases. For the fuzzy observer's stability and design, they considered measurable and unmeasurable premise variables for continuous and

Received (September 3, 2017), Review Result (December 14, 2017), Accepted (December 27, 2017)

${ }^{*}$ Corresponding Author 
discrete TS fuzzy systems, they used in all steps of design the LMI tools to prove and to express the stability conditions.

$\mathrm{X}$. Ma et al. [2] designed a fuzzy observer based on common quadratic Laypunov function, in this case they show that the separation principle does hold for the augmented TS fuzzy system, which means that the design procedure of fuzzy observer and fuzzy controller can be done independently.

A survey of model-based fuzzy theory is considered in [13] with citation of important papers in fuzzy modeling, fuzzy control, different fuzzy observers design.

Among papers who considered unmeasurable premise variable, we can quote the works of Bergsten et al. [10] which designed fuzzy sliding mode observer with local affine models, some works of the same case are reported in [9][6]

P. BERGSTEN, and R. PALM proposed a Thau-Luenberger TS fuzzy observer with stability analysis and design procedure [7]

Author in [3] presented a new stability condition for TS fuzzy observer with unmeasurable premise variables.

Paper [16] proposed an observer-based controller for nonlinear system represented by TS fuzzy models using unmeasurable premise variables, the proposed TS structure reduces the number of rules in TS models as well as analysis complexity.

Authors in [17] considered a fuzzy-model-based observer-controller using an unmeasurable premise variable, they relax the stability conditions by considering the membership functions information's in stability conditions.

Lot of authors in literature considered two states for the premise variable [1] [3] [5], but they do not be interested by all possible cases when we use the same premise variable (measurable or not) [14] [15], or we use different premise variable for TS fuzzy model and TS fuzzy observer.

In this paper we try to give the different possible cases, and we do a comment about Tanaka et al. paper [1] which contain fault in the theorem 11 of stability of augmented system when they use the unmeasurable premise variable.

This paper is organized in 6 sections as following: an introduction which presents some previous works, section II for TS fuzzy system presentation, section III describes the problem asked, in section IV we show the first case of measurable premise variable, and the second case of unmeasurable premise variable is presented section $\mathrm{V}$, and we finish this papers by a conclusion.

\section{TS Fuzzy Modeling}

The main idea of TS fuzzy modeling is to represent a nonlinear system with a set of elementary local models which describe the nonlinear system in different operating range, this local models can be linear or affine models.

To characterize the overall dynamics of nonlinear system, the local models are aggregated by fuzzy membership function, such as every local model represent a consequence of fuzzy rule given as:

IF $\theta(t)$ is $M_{i}$ THEN:

Such as:

$$
\dot{x}_{i}(t)=A_{i} x(t)+B_{i} u(t)
$$

$\theta(t)$ : is the premise variable $M_{i}$ : is the $i^{\text {th }}$ fuzzy set.

$x(t) \in R^{n}$ : is the state vector. $u(t) \in R^{n}$ : Input vector.

$A_{i} \in R^{n \times n}$ : The local state matrix. $B_{i} \in R^{n \times m}$ : The local input matrix.

The rule condition (IF $\theta(t)$ is $M_{i}$ ) defines the local operating point condition, and the overall TS fuzzy system is given as:

$$
\dot{x}(t)=\sum_{1}^{r} \omega_{i}(\theta(t))\left(A_{i} x(t)+B_{i} u(t)\right)
$$


$\omega_{i}(\theta(t))$ : is the grade of the $i^{t h}$ fuzzy set membership function.

With:

$$
0<\omega_{i}(\theta(t))<1 \quad \text { and } \quad \sum_{i=1}^{r} \omega_{i}(\theta(t))=1
$$

$r$ : represent fuzzy rule number which is also the local linear models number.

Theorem 1: [8]

The open loop of TS fuzzy system $(2)(u(t)=0)$ is globally asymptotically stable if there is a common positive definite matrix $P$ such as the following LMI are checked:

$$
\mathrm{A}_{\mathrm{i}}^{\mathrm{T}} \mathrm{P}+\mathrm{PA}_{\mathrm{i}}<0 \forall i \in\{1, \ldots, \mathrm{r}\}
$$

\section{Problem Description and Premise Variable Choice}

In this paper our aims are the stabilization of the TS fuzzy system given as:

With PDC controller given as:

$$
\dot{x}(t)=\sum_{1}^{r} \omega_{i}(\theta(t))\left(A_{i} x(t)+B_{i} u(t)\right)
$$

$$
u(t)=\sum_{1}^{r} \omega_{j}(\theta(t))\left(-K_{j} \hat{x}(t)\right)
$$

This PDC controller depends on the state vector which is not measurable in general, and requires an observer to be estimated.

For this raison we design a TS fuzzy observer given as:

$$
\dot{\hat{x}}(t)=\sum_{1}^{r} \omega_{i}(\theta(t))\left(A_{i} \hat{x}(t)+B_{i} u(t)+L_{i}(y(t)-\hat{y}(t))\right.
$$

For the design of the controller/ observer gains we consider the augmented system defined as:

Which yields

$$
X_{a}(t)=\left(\begin{array}{l}
x(t) \\
e(t)
\end{array}\right)
$$

\subsection{Existed (Classical) Classification}

$$
\dot{X}_{a}(t)=\left(\begin{array}{c}
\dot{x}(t) \\
\dot{e}(t)
\end{array}\right)
$$

In the literature, authors consider two cases of premise variable during the design of TS fuzzy model with a fuzzy observer, they consider a measurable and an unmeasurable cases as following:

A. measurable premise variable:

In this case, they use a measurable premise variable for:

- TS fuzzy model

- TS fuzzy observer

\section{B. unmeasurable premise variable:}

When authors mention the unmeasurable premise variable case [1] [3] [5] [9][10], they use different premise variable as following:

- A measurable premise variable for TS fuzzy model.

- An unmeasurable premise variable for TS fuzzy observer. 


\subsection{Our (New) Classification}

In this paragraph, we present a new consideration for the premise variable cases, we use the term "nature" for the premise variable to indicate its measurability (either measurable or unmeasurable). Our classification is given as following:

A. same nature of premise variable ( 2 possibilities):

$>$ the same measurable premise variable for:

- The TS fuzzy model

- The fuzzy observer

$>$ the same unmeasurable premise variable for :

- The TS fuzzy model

- The fuzzy observer

B. different nature of premise variable :

- A measurable premise variable for the TS fuzzy model.

- An unmeasurable premise variable for the fuzzy observer.

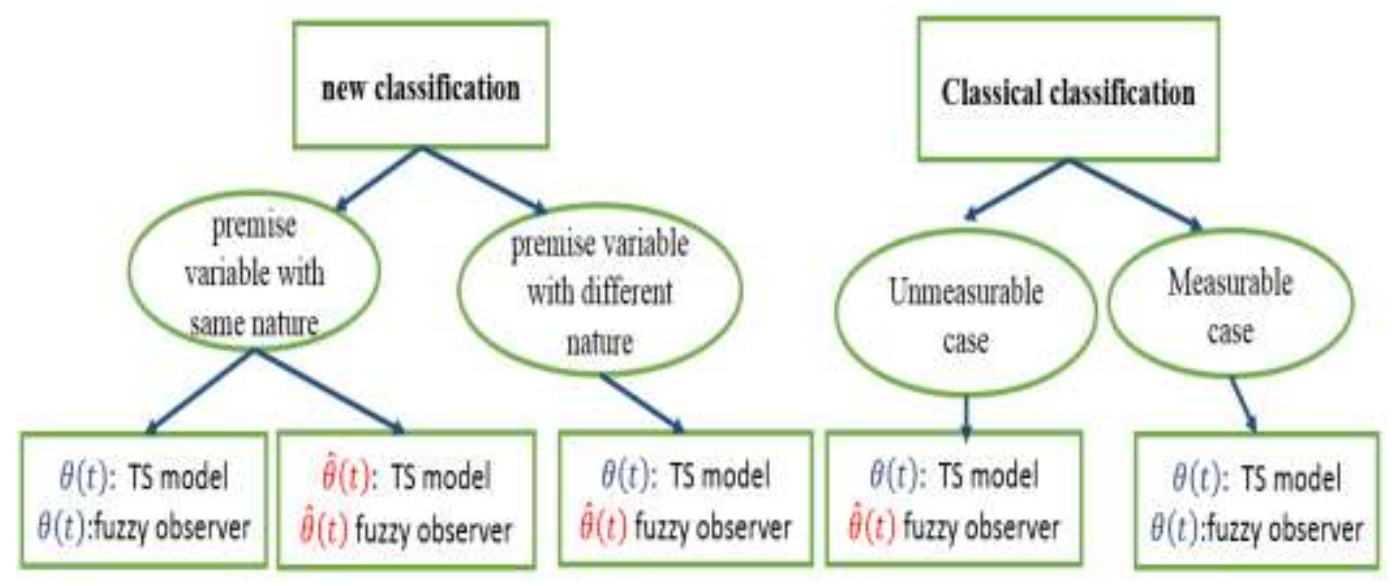

Figure 1. Classifications of Premise Variable Utilization

According to our proposition, there are two overall cases, which are:

- Same premise variable (measurable or unmeasurable) for system TS model and fuzzy observer.

- Different premise variable (one measurable and the other unmeasurable) for system TS model and fuzzy observer.

We prove in the next sections that the use of the same or different nature of premise variable for the fuzzy model (state) and fuzzy observer is the real factor to change the procedure of PDC and observer design.

\section{Case one: The Same Nature of Premise Variable both Measurable or both Unmeasurable}

In all next steps we design fuzzy LUENBERGER observers, we remember its equation in linear case as follows:

$$
\dot{\hat{x}}(t)=A \hat{x}(t)+B u(t)+L(y(t)-\hat{y}(t))
$$

Where the estimation error is given as:

$$
e(t)=x(t)-\hat{x}(t) \Rightarrow \dot{e}(t)=(A-L C) e(t)
$$


The above observer is asymptotically stable if the matrix $(A-L C)$ have all eigenvalues in the negative demi-plan.

\subsection{First Possibility: (Measurable \& Measurable $\theta(t)$ )}

We consider a measurable premise variable $\theta(t)$ for TS fuzzy model, for PDC controller and for the fuzzy observer such as:

We consider a TS fuzzy model given as:

$$
\dot{x}(t)=\sum_{1}^{r} \omega_{i}(\theta(t))\left(A_{i} x(t)+B_{i} u(t)\right)
$$

And the fuzzy observer equation is:

$$
\begin{gathered}
\dot{\hat{x}}(t)=\sum_{1}^{r} \omega_{i}(\theta(t))\left(A_{i} \hat{x}(t)+B_{i} u(t)+L_{i}(y(t)-\hat{y}(t))\right) \\
\hat{y}(t)=C \hat{x}(t)
\end{gathered}
$$

With $C=\left[\begin{array}{ll}0 & 1\end{array}\right]$ : is the output matrix.

$\hat{y}(t)$ : is the estimated output.

$L_{i}$ : are the local observer gain vectors.

The common PDC controller depends on the estimated state vector, it is given as:

The closed loop:

$$
u(t)=\sum_{1}^{r} \omega_{j}(\theta(t))\left(-K_{j} \hat{x}(t)\right)
$$

We have:

$$
\dot{x}(t)=\sum_{1}^{r} \omega_{i}(\theta(t))\left(A_{i} x(t)+B_{i} \sum_{1}^{r} \omega_{j}(\theta(t))\left(-K_{j} \hat{x}(t)\right)\right)
$$

$$
e(t)=x(t)-\hat{x}(t) \Rightarrow \hat{x}(t)=x(t)-e(t)
$$

The equation (16) will be given as:

$$
\dot{x}(t)=\sum_{1}^{r} \omega_{i}(\theta(t))\left(A_{i} x(t)-B_{i} \sum_{j=1}^{r} \omega_{j}(\theta(t)) K_{j}(x(t)-e(t))\right)
$$

By expansion of the equation (18) we will have finally:

$$
\begin{aligned}
\dot{x}(t)=\sum_{i=1}^{r} \sum_{j=1}^{r} \omega_{i}(\theta(t)) \omega_{j}(\theta(t))\left(A_{i}-B_{i} K_{j}\right) \boldsymbol{x}(\boldsymbol{t}) \\
+\sum_{i=1}^{r} \sum_{j=1}^{r} \omega_{i}(\theta(t)) \omega_{j}(\theta(t))\left(B_{i} K_{j}\right) \boldsymbol{e}(\boldsymbol{t})
\end{aligned}
$$

\section{The estimation error dynamics:}

We note that in all previous equations we use the same $\theta(t)$ the estimation error is considered as:

$$
e(t)=x(t)-\hat{x}(t) \Rightarrow \dot{e}(t)=\dot{x}(t)-\dot{\hat{x}}(t)
$$




$$
\begin{aligned}
& \dot{e}(t)=\sum_{i=1}^{r} \omega_{i}(\theta(t))\left(A_{i} x(t)+B_{i} u(t)\right) \\
&-\sum_{i=1}^{r} \omega_{i}(\theta(t))\left(\left(A_{i} \hat{x}(t)+B_{i} u(t)\right)+L_{i}(y(t)-\hat{y}(t))\right) \\
& \dot{e}(t)=\sum_{i=1}^{r} \omega_{i}(\theta(t))\left(A_{i}-L_{i} C\right)(x(t)-\hat{x}(t))
\end{aligned}
$$

The estimation error dynamics is given as:

$$
\dot{e}(t)=\sum_{i=1}^{r} \omega_{i}(\theta(t))\left(A_{i}-L_{i} C\right) e(t)
$$

Note: to get the fuzzy observer for the system (2), it must have all pair $\left(A_{i}, C\right)$ is observable.

The augmented system:

From the equation (19) and (23), we consider the augmented system:

$$
\begin{gathered}
\dot{\boldsymbol{X}}_{\boldsymbol{a}}(\boldsymbol{t})=\left(\begin{array}{c}
\dot{x}(t) \\
\dot{e}(t)
\end{array}\right) \\
\dot{\boldsymbol{X}}_{\boldsymbol{a}}(\boldsymbol{t})=\sum_{i=1}^{r} \sum_{j=1}^{r} \omega_{i}(\theta(t)) \omega_{j}(\theta(t)) \boldsymbol{G}_{\boldsymbol{i j}}\left(\begin{array}{c}
x(t) \\
e(t)
\end{array}\right) \\
\boldsymbol{G}_{\boldsymbol{i} \boldsymbol{j}}=\left(\begin{array}{cc}
A_{i}-B_{i} K_{j} & B_{i} K_{j} \\
0 & A_{i}-L_{i} C
\end{array}\right) \\
\dot{\boldsymbol{X}}_{\boldsymbol{a}}(\boldsymbol{t})=\sum_{i=1}^{r} \omega_{i}(\theta(t))^{2} \boldsymbol{G}_{\boldsymbol{i} \boldsymbol{i}} \boldsymbol{X}_{\boldsymbol{a}}(\boldsymbol{t})+\mathbf{2} \sum_{i=1}^{r} \sum_{i<j}^{r} \omega_{i}(\theta(t)) \omega_{j}(\theta(t))\left(\frac{\boldsymbol{G}_{\boldsymbol{i j}}+\boldsymbol{G}_{\boldsymbol{j} \boldsymbol{i}}}{\mathbf{2}}\right) \boldsymbol{X}_{\boldsymbol{a}}(\boldsymbol{t})
\end{gathered}
$$

Theorem 2:

The augmented system $X_{a}(t)=[x(t) ; e(t)]$ modeled by (27) is asymptotically stable if there exists a positive definite matrix $P$ such as the following conditions hold $i, j=1, \ldots, r$ :

$$
\begin{aligned}
& \mathrm{G}_{i i}{ }^{T} \boldsymbol{P}+\boldsymbol{P G}_{i i}<0 \quad \forall \boldsymbol{i} \\
& \left(\frac{G_{i j}+G_{j i}}{2}\right)^{T} P+P\left(\frac{G_{i j}+G_{j i}}{2}\right)<0 \quad \forall i<j
\end{aligned}
$$

\section{Result:}

From the theorem 2 we conclude that the design of controller and observer can be done separately, in other word these is no dependence between controller and observer design.

Such as the estimation error do not depend on the state vector.

\section{Design Procedure of the fuzzy observer without PDC controller gains:}

Theorem3: [1, 2, And 4] the fuzzy observer described by equation (13) and (14) is asymptotically stable if there exists a common positive definite matrix $P$ such that:

$$
\left(A_{i}-L_{i} C\right)^{T} P+P\left(A_{i}-L_{i} C\right)<0 i \in 1,2, \ldots, r
$$


The observer response must be faster than the system dynamic, for this raison we have to place the observer eigenvalues values precisely. Let $S(\alpha, \beta)$ the region delimited by the circle with the center $(0 ; 0)$ radius $\beta$ and the strip with real part smaller than $-\alpha$.

Corollary [4]: All local observers in the fuzzy observer have their eigenvalues in the region $S(\alpha, \beta)$, if there exists a common positive definite matrix $\mathrm{P}$ such that:

$$
\begin{gathered}
\left(\begin{array}{cc}
-\beta P & \left(A_{i}-L_{i} C\right)^{T} P \\
P\left(A_{i}-L_{i} C\right) & -\beta P
\end{array}\right)<0 \\
\left(A_{i}-L_{i} C\right)^{T} P+P\left(A_{i}-L_{i} C\right)+2 \alpha P<0
\end{gathered}
$$

To resolve these optimization problem we have to transform it to LMIs form such as; We remind that $(A B)^{T}=B^{T} A^{T}$.

$$
\begin{gathered}
\left(\begin{array}{cc}
-\beta P & A_{i}{ }^{T} P-C^{T} L_{i}{ }^{T} P \\
P A_{i}-P L_{i} C & -\beta P
\end{array}\right)<0 \\
A_{i}{ }^{T} P-C^{T} L_{i}{ }^{T} P+P A_{i}-P L_{i} C+2 \alpha P<0
\end{gathered}
$$

We make the following changing variable

$$
P L_{i}=M_{i}
$$

Which yield the following LMIs in $P$ and $M_{i}$ :

$$
\begin{array}{r}
\left(\begin{array}{cc}
-\beta P & A_{i}{ }^{T} P-C^{T} M_{i}{ }^{T} \\
P A_{i}-M_{i} C & -\beta P
\end{array}\right)<0 \\
A_{i}{ }^{T} P-C^{T} M_{i}{ }^{T}+P A_{i}-M_{i} C+2 \alpha P<0
\end{array}
$$

Finally, the observer gains will be given as:

$$
L_{i}=P^{-1} M_{i}
$$

\subsection{Second Possibility: (unmeasurable \& unmeasurable $\widehat{\theta}(t)$ )}

In this case we consider an unmeasurable premise variable $\hat{\theta}(t)$ for TS fuzzy model given as:

$$
\dot{x}(t)=\sum_{i=1}^{r} \omega_{i}(\hat{\theta}(t))\left(A_{i} x(t)+B_{i} u(t)\right)
$$

An unmeasurable premise variable $\hat{\theta}(t)$ For the PDC controller:

$$
u(t)=\sum_{j=1}^{r} \omega_{j}(\hat{\theta}(t))\left(-K_{j} \hat{x}(t)\right)
$$

And an unmeasurable premise variable $\hat{\theta}(t)$ for the fuzzy observer is given as;

$$
\dot{\hat{x}}(t)=\sum_{i=1}^{r} \omega_{i}(\hat{\theta}(t))\left(A_{i} \hat{x}(t)+B_{i} u(t)+L_{i}(y(t)-\hat{y}(t))\right.
$$

We note that the unmeasurable premise variable $\hat{\theta}(t)$ is estimated by the fuzzy observer. 
From a stability viewpoint nothing has changed when we replace the measurable with unmeasurable premise variable such as; the estimation error dynamics will be given as:

$$
\dot{e}(t)=\sum_{i=1}^{r} \omega_{i}(\hat{\theta}(t))\left(A_{i}-L_{i} C\right) e(t)
$$

\section{Result:}

The observers $(13,14),(41,14)$ have the same stability condition of theorem 2 if we use the same nature of measurable or unmeasurable premise variable for both TS fuzzy system and TS fuzzy observer.

\section{Case Two: Different Nature of Premise Variables}

For this section we consider a measurable premise variable $\theta(t)$ for TS fuzzy model, and we use an unmeasurable premise variable $\hat{\theta}(t)$ for the fuzzy observer such as:

The TS fuzzy model will be given as:

$$
\dot{x}(t)=\sum_{i=1}^{r} \omega_{i}(\theta(t))\left(A_{i} x(t)+B_{i} u(t)\right)
$$

The PDC controller depends on the estimated state, then it depends on an unmeasurable premise variable $\hat{\theta}(t)$ as following:

$$
u(t)=\sum_{k=1}^{r} \omega_{k}(\hat{\theta}(t))\left(-K_{k} \hat{x}(t)\right)
$$

The closed loop equation will be given as:

$$
\dot{x}(t)=\sum_{i=1}^{r} \omega_{i}(\theta(t))\left(A_{i} x(t)-B_{i} \sum_{k=1}^{r} \omega_{k}(\hat{\theta}(t)) K_{k} \hat{x}(t)\right)
$$

The fuzzy observer will be (precedent equation) given as:

$$
\dot{\hat{x}}(t)=\sum_{j=1}^{r} \omega_{j}(\hat{\theta}(t))\left(A_{j} \hat{x}(t)+B_{j} u(t)+L_{j}(y(t)-\hat{y}(t))\right.
$$

We define the estimation error as:

$$
e(t)=x(t)-\hat{x}(t)=>\hat{x}(t)=x(t)-e(t)
$$

A. The closed loop equation:

$$
\dot{x}(t)=\sum_{i=1}^{r} \omega_{i}(\theta(t))\left(A_{i} x(t)-B_{i} \sum_{k=1}^{r} \omega_{k}(\hat{\theta}(t)) K_{k}(x(t)-e(t))\right.
$$

In closed loop, the state dynamics with a fuzzy observer will be given as

$$
\begin{aligned}
\dot{x}(t) & =\sum_{i=1}^{r} \sum_{k=1}^{r} \omega_{i}(\theta(t)) \omega_{k}(\hat{\theta}(t))\left(A_{i}-B_{i} K_{k}\right) \boldsymbol{x}(\boldsymbol{t}) \\
& +\sum_{i=1}^{r} \sum_{k=1}^{r} \omega_{i}(\theta(t)) \omega_{k}(\hat{\theta}(t))\left(B_{i} K_{k}\right) \boldsymbol{e}(\boldsymbol{t})
\end{aligned}
$$

\section{B. The Estimation Error Dynamics}

The estimation error dynamics will be:

$$
\dot{e}(t)=\sum_{i=1}^{r} \omega_{i}(\theta(t))\left(A_{i} x(t)+B_{i} u(t)\right)
$$




$$
\begin{array}{r}
-\sum_{j=1}^{r} \omega_{j}(\hat{\theta}(t))\left(A_{j} \hat{x}(t)+B_{j} u(t)+L_{j}(y(t)-\hat{y}(t))\right. \\
\dot{e}(t)=\sum_{i=1}^{r} \omega_{i}(\theta(t)) A_{i} x(t)-\sum_{j=1}^{r} \omega_{j}(\hat{\theta}(t)) A_{j} \hat{x}(t)+\sum_{i=1}^{r} \omega_{i}(\theta(t)) B_{i} u(t) \\
-\sum_{j=1}^{r} \omega_{j}(\hat{\theta}(t)) B_{j} u(t)-\sum_{j=1}^{r} \omega_{j}(\hat{\theta}(t)) L_{j} \operatorname{Ce}(t)
\end{array}
$$

By incorporating the convex sum in the equation (51):

$$
\sum_{i=1}^{r} \omega_{i}(\theta(t))=1 \text { and } \sum_{j=1}^{r} \omega_{k}(\hat{\theta}(t))=1
$$

And substituting $\hat{x}(t)=x(t)-e(t)$ then:

$$
\begin{aligned}
& \dot{e}(t)=\sum_{i=1}^{r} \sum_{j=1}^{r} \omega_{i}(\theta(t)) \omega_{j}(\hat{\theta}(t)) A_{i} x(t)-\sum_{i=1}^{r} \sum_{j=1}^{r} \omega_{i}(\theta(t)) \omega_{j}(\hat{\theta}(t)) A_{j} x(t) \\
&+\sum_{i=1}^{r} \sum_{j=1}^{r} \omega_{i}(\theta(t)) \omega_{j}(\hat{\theta}(t)) B_{i} u(t)-\sum_{i=1}^{r} \sum_{j=1}^{r} \omega_{i}(\theta(t)) \omega_{j}(\hat{\theta}(t)) B_{j} u(t) \\
&+\sum_{j=1}^{r} \omega_{j}(\hat{\theta}(t)) A_{j} e(t)-\sum_{j=1}^{r} \omega_{j}(\hat{\theta}(t)) L_{j} C e(t) \\
& \dot{e}(t)=\sum_{i=1}^{r} \sum_{j=1}^{r} \omega_{i}(\theta(t)) \omega_{j}(\hat{\theta}(t))\left(A_{i}-A_{j}\right) x(t)+\sum_{i=1}^{r} \sum_{j=1}^{r} \omega_{i}(\theta(t)) \omega_{j}(\hat{\theta}(t))\left(B_{i}-B_{j}\right) u(t) \\
&+\sum_{j=1}^{r} \omega_{j}(\hat{\theta}(t))\left(A_{j}-L_{j} C\right) e(t)
\end{aligned}
$$

By substituting the controller given by the equation (45) we will have:

$$
\begin{aligned}
& \dot{e}(t)=\sum_{i=1}^{r} \sum_{j=1}^{r} \omega_{i}(\theta(t)) \omega_{j}(\hat{\theta}(t))\left(A_{i}-A_{j}\right) x(t)+\sum_{j=1}^{r} \omega_{j}(\hat{\theta}(t))\left(A_{j}-L_{j} C\right) e(t) \\
& +\sum_{i=1}^{r} \sum_{j=1}^{r} \omega_{i}(\theta(t)) \omega_{j}(\hat{\theta}(t))\left(B_{i}-B_{j}\right) \sum_{k=1}^{r} \omega_{k}(\hat{\theta}(t))\left(-K_{k} \hat{x}(t)\right)
\end{aligned}
$$

The estimated state is given as: $\hat{x}(t)=x(t)-e(t)$

$$
\begin{gathered}
\dot{e}(t)=\sum_{i=1}^{r} \sum_{j=1}^{r} \omega_{i}(\theta(t)) \omega_{j}(\hat{\theta}(t))\left(A_{i}-A_{j}\right) x(t)+\sum_{j=1}^{r} \omega_{j}(\hat{\theta}(t))\left(A_{j}-L_{j} C\right) e(t) \\
+\sum_{i=1}^{r} \sum_{j=1}^{r} \omega_{i}(\theta(t)) \omega_{j}(\hat{\theta}(t))\left(B_{i}-B_{j}\right) \sum_{k=1}^{r} \omega_{k}(\hat{\theta}(t))\left(-K_{k}(x(t)-e(t))\right) \\
\dot{e}(t)=\sum_{i=1}^{r} \sum_{j=1}^{r} \omega_{i}(\theta(t)) \omega_{j}(\hat{\theta}(t))\left(A_{i}-A_{j}\right) x(t)+\sum_{j=1}^{r} \omega_{j}(\hat{\theta}(t))\left(A_{j}-L_{j} C\right) e(t) \\
\quad-\sum_{i=1}^{r} \sum_{j=1}^{r} \sum_{k=1}^{r} \omega_{i}(\theta(t)) \omega_{j}(\hat{\theta}(t)) \omega_{k}(\hat{\theta}(t))\left(B_{i}-B_{j}\right) K_{k} x(t)
\end{gathered}
$$


$+\sum_{i=1}^{r} \sum_{j=1}^{r} \sum_{k=1}^{r} \omega_{i}(\theta(t)) \omega_{j}(\hat{\theta}(t)) \omega_{k}(\hat{\theta}(t))\left(B_{i}-B_{j}\right) K_{k} e(t)$

By incorporating the convex sum:

$$
\sum_{i=1}^{r} \omega_{i}(\theta(t))=1 \text { and } \sum_{j=1}^{r} \omega_{k}(\hat{\theta}(t))=1
$$

In the equation of the estimation error dynamics, we will get:

$$
\begin{array}{r}
\dot{e}(t)=\sum_{i=1}^{r} \sum_{j=1}^{r} \sum_{k=1}^{r} \omega_{i}(\theta(t)) \omega_{j}(\hat{\theta}(t)) \omega_{k}(\hat{\theta}(t))\left(A_{i}-A_{j}\right) \boldsymbol{x}(\boldsymbol{t}) \\
-\sum_{i=1}^{r} \sum_{j=1}^{r} \sum_{k=1}^{r} \omega_{i}(\theta(t)) \omega_{j}(\hat{\theta}(t)) \omega_{k}(\hat{\theta}(t))\left(B_{i}-B_{j}\right) K_{k} \boldsymbol{x}(\boldsymbol{t}) \\
+\sum_{i=1}^{r} \sum_{j=1}^{r} \sum_{k=1}^{r} \omega_{i}(\theta(t)) \omega_{j}(\hat{\theta}(t)) \omega_{k}(\hat{\theta}(t))\left(A_{j}-L_{j} C\right) \boldsymbol{e}(\boldsymbol{t}) \\
+\sum_{i=1}^{r} \sum_{j=1}^{r} \sum_{k=1}^{r} \omega_{i}(\theta(t)) \omega_{j}(\hat{\theta}(t)) \omega_{k}(\hat{\theta}(t))\left(B_{i}-B_{j}\right) K_{k} \boldsymbol{e}(\boldsymbol{t})
\end{array}
$$

The final equation will simplified as:

$$
\begin{aligned}
\dot{e}(t)=\sum_{i=1}^{r} \sum_{j=1}^{r} \sum_{k=1}^{r}\left(\omega_{i}(\theta(t)) \omega_{j}(\hat{\theta}(t))\right) \omega_{k}(\hat{\theta}(t))\left[\boldsymbol{A}_{\boldsymbol{i}}-\boldsymbol{A}_{\boldsymbol{j}}-\left(\boldsymbol{B}_{\boldsymbol{i}}-\boldsymbol{B}_{\boldsymbol{j}}\right) \boldsymbol{K}_{\boldsymbol{k}}\right] \boldsymbol{x}(\boldsymbol{t}) \\
+\sum_{i=1}^{r} \sum_{j=1}^{r} \sum_{k=1}^{r} \omega_{i}(\theta(t)) \omega_{j}(\hat{\theta}(t)) \omega_{k}(\hat{\theta}(t))\left[\left(\boldsymbol{A}_{\boldsymbol{j}}-\boldsymbol{L}_{\boldsymbol{j}} \boldsymbol{C}\right)\right. \\
\left.+\left(\boldsymbol{B}_{\boldsymbol{i}}-\boldsymbol{B}_{\boldsymbol{j}}\right) \boldsymbol{K}_{\boldsymbol{k}}\right] \boldsymbol{e}(\boldsymbol{t})
\end{aligned}
$$

\section{The Augmented System:}

We incorporate the convex sum (59) in (50) (equation of the state dynamics):

$$
\begin{gathered}
\sum_{j=1}^{r} \omega_{j}(\hat{\theta}(t))=1 \\
\dot{x}(t)=\sum_{i=1}^{r} \sum_{j=1}^{r} \sum_{k=1}^{r} \omega_{i}(\theta(t)) \omega_{j}(\hat{\theta}(t)) \omega_{k}(\hat{\theta}(t))\left(A_{i}-B_{i} K_{k}\right) x(t) \\
+\sum_{i=1}^{r} \sum_{j=1}^{r} \sum_{k=1}^{r} \omega_{i}(\theta(t)) \omega_{j}(\hat{\theta}(t)) \omega_{k}(\hat{\theta}(t))\left(B_{i} K_{k}\right) e(t)
\end{gathered}
$$

Finally we have obtain the augmented system with the state dynamics $\dot{\boldsymbol{x}}(\boldsymbol{t})$ and the estimation error dynamicse $(\boldsymbol{t})$ :

$$
\left(\begin{array}{c}
\dot{x}(t) \\
\dot{e}(t)
\end{array}\right)=\sum_{i=1}^{r} \sum_{j=1}^{r} \sum_{k=1}^{r} \omega_{i}(\theta(t)) \omega_{j}(\hat{\theta}(t)) \omega_{k}(\hat{\theta}(t)) \boldsymbol{G}_{\boldsymbol{i j k}}\left(\begin{array}{l}
x(t) \\
e(t)
\end{array}\right)
$$

We take:

$$
\begin{gathered}
\dot{\boldsymbol{X}}_{\boldsymbol{a}}(\boldsymbol{t})=\left(\begin{array}{c}
\dot{x}(t) \\
\dot{e}(t)
\end{array}\right) \\
\boldsymbol{G}_{\boldsymbol{i j k} \boldsymbol{k}}=\left(\begin{array}{cc}
A_{i}-B_{i} K_{k} & B_{i} K_{k} \\
A_{i}-A_{j}-\left(B_{i}-B_{j}\right) K_{k} & A_{j}-L_{j} C+\left(B_{i}-B_{j}\right) K_{k}
\end{array}\right)
\end{gathered}
$$




$$
\begin{aligned}
\dot{\boldsymbol{X}}_{\boldsymbol{a}}(\boldsymbol{t})=\sum_{i=1}^{r} \sum_{j=1}^{r} \sum_{k=1}^{r} \omega_{i}(\theta(t)) \omega_{j}(\hat{\theta}(t)) \omega_{k}(\hat{\theta}(t)) \boldsymbol{G}_{\boldsymbol{i} \boldsymbol{j} \boldsymbol{k}} \boldsymbol{X}_{\boldsymbol{a}}(\boldsymbol{t}) \\
\dot{\boldsymbol{X}}_{\boldsymbol{a}}(\boldsymbol{t})=\sum_{i=1}^{r} \sum_{j=1}^{r} \omega_{i}(\theta(t)) \omega_{j}(\hat{\theta}(t)) \omega_{i}(\hat{\theta}(t)) \boldsymbol{G}_{\boldsymbol{i j} \boldsymbol{i}} \boldsymbol{X}_{\boldsymbol{a}}(\boldsymbol{t}) \\
+\mathbf{2} \sum_{i=1}^{r} \sum_{j=1}^{r} \sum_{i<k}^{r} \omega_{i}(\theta(t)) \omega_{j}(\hat{\theta}(t)) \omega_{k}(\hat{\theta}(t))\left(\frac{\boldsymbol{G}_{\boldsymbol{i} \boldsymbol{j} \boldsymbol{k}}+\boldsymbol{G}_{\boldsymbol{k} \boldsymbol{j} \boldsymbol{i}}}{\mathbf{2}}\right) \boldsymbol{X}_{\boldsymbol{a}}(\boldsymbol{t})
\end{aligned}
$$

PROPOSITION 1: The stability conditions of the augmented system:

The augmented system $X_{a}(t)=[x(t) ; e(t)]$ modeled by $(65)$ is asymptotically stable if there exists a positive definite matrix $P$ such as the following condition hold for $i, j, k=1, \ldots, r$ :

$$
\begin{array}{cr}
\mathbf{G}_{i j i}{ }^{T} P+P_{G_{i j i}}<0 & \forall i, \forall j \\
\left(\frac{G_{i j k}+G_{k j i}}{2}\right)^{T} P+P\left(\frac{G_{i j k}+G_{k j i}}{2}\right)<0 & \forall j, i<k
\end{array}
$$

Comment:

This proposition is mentioned in form of:

Theorem 11-CFS (Continuous Fuzzy System) in the paper [1], and in form of theorem 20 of the book "FUZZY CONTROL SYSTEMS DESIGN AND ANALYSIS" both of Kazuo Tanaka and al. But mentioned theorems have a fault in the indexation for $\boldsymbol{j}, \boldsymbol{i}$ and s such as:

The stability condition must hold on $\forall \boldsymbol{j}, \boldsymbol{i}<\boldsymbol{s}$ and not for $\forall \boldsymbol{i}, \boldsymbol{j}<\boldsymbol{s}$ which mean that the permutation of index is done between $\boldsymbol{i}$ and $\boldsymbol{s}\left(\boldsymbol{G}_{\boldsymbol{i j} \boldsymbol{s}}, \boldsymbol{G}_{\boldsymbol{s j i}}\right)$ and not between j and $s\left(G_{i j s}, G_{i s j}\right)$

\section{Result:}

From the proposition (1), we conclude that the design of controller and observer cannot be done separately, because these is dependence terms between controller and observer design.

\section{Special case $B_{i}=B$ :}

$$
\begin{aligned}
& \text { If we have } B_{i}=B \text { the augmented system will be: } \\
& \dot{\boldsymbol{X}}_{\boldsymbol{a}}(\boldsymbol{t})=\sum_{i=1}^{r} \sum_{j=1}^{r} \sum_{k=1}^{r} \omega_{i}(\theta(t)) \omega_{j}(\hat{\theta}(t)) \omega_{k}(\hat{\theta}(t)) \boldsymbol{G}_{\boldsymbol{i} \boldsymbol{j} \boldsymbol{k}}\left(\begin{array}{c}
x(t) \\
e(t)
\end{array}\right) \\
& \qquad \boldsymbol{G}_{\boldsymbol{i} \boldsymbol{j} \boldsymbol{k}}=\left(\begin{array}{cc}
\boldsymbol{A}_{\boldsymbol{i}}-\boldsymbol{B K}_{\boldsymbol{k}} & \boldsymbol{B} \boldsymbol{K}_{\boldsymbol{k}} \\
\boldsymbol{A}_{\boldsymbol{i}}-\boldsymbol{A}_{\boldsymbol{j}} & \boldsymbol{A}_{\boldsymbol{j}}-\boldsymbol{L}_{\boldsymbol{j}} \boldsymbol{C}
\end{array}\right)
\end{aligned}
$$

Linearization of the first condition of proposition (1) when $B_{i}=B$ :

The next step we expanse only the first stability condition of our proposition to have its LMI form, and the same procedure is valid for the second condition:

$$
\left(\begin{array}{cc}
A_{i}-B K_{k} & B K_{k} \\
A_{i}-A_{j} & A_{j}-L_{j} C
\end{array}\right)^{T} P+P\left(\begin{array}{cc}
A_{i}-B K_{k} & B K_{k} \\
A_{i}-A_{j} & A_{j}-L_{j} C
\end{array}\right)<0
$$

We note that this equality is not an LMI, so we must to make a variable change to transform it to an LMI.

We take a new variable $\boldsymbol{X}=\boldsymbol{P}^{-\mathbf{1}}$, by multiplying left and right the previous equality (76) we will have:

$$
X\left(\begin{array}{cc}
{A_{i}}^{T}-K_{i}{ }^{T} B^{T} & A_{i}{ }^{T}-A_{j}{ }^{T} \\
K_{i}{ }^{T} B^{T} & A_{j}{ }^{T}-C^{T} L_{j}{ }^{T}
\end{array}\right)+\left(\begin{array}{cc}
A_{i}-B K_{i} & B K_{i} \\
A_{i}-A_{j} & A_{j}-L_{j} C
\end{array}\right) X<0
$$




$$
\left(\begin{array}{cc}
X A_{i}{ }^{T}-X K_{i}{ }^{T} B^{T} & X\left(A_{i}{ }^{T}-A_{j}{ }^{T}\right) \\
X K_{i}{ }^{T} B^{T} & X A_{j}{ }^{T}-X C^{T} L_{j}{ }^{T}
\end{array}\right)+\left(\begin{array}{cc}
A_{i} X-B K_{i} X & B K_{i} X \\
\left(A_{i}-A_{j}\right) X & A_{j} X-L_{j} C X
\end{array}\right)<0
$$

The take $\boldsymbol{M}_{\boldsymbol{i}}=\boldsymbol{K}_{\boldsymbol{i}} \boldsymbol{X}$ and $\boldsymbol{N}_{\boldsymbol{j}}=\boldsymbol{L}_{\boldsymbol{j}} \boldsymbol{C X}$, it yields the following LMI in $\boldsymbol{X}$ and $\boldsymbol{M}_{\boldsymbol{i}}$ :

$$
\left(\begin{array}{cc}
X A_{i}{ }^{T}-M_{i}{ }^{T} B^{T}+A_{i} X-M_{i} X & X\left(A_{i}{ }^{T}-A_{j}{ }^{T}\right)+B M_{i} \\
M_{i}{ }^{T} B^{T}+\left(A_{i}-A_{j}\right) X & X A_{j}{ }^{T}-N_{j}{ }^{T}+A_{j} X-N_{j}
\end{array}\right)<0
$$

You can do the same calculus procedure for the general case $B_{i} \neq B$ and for the second stability condition to have its LMI form.

\section{Conclusion}

The stability analysis and design procedure of the augmented TS fuzzy systems (state and observer) requires some consideration about the nature of premise variable either measurable or unmeasurable, which is related to the dependence of premise variable to the state vector, according to this relationship we can distinguish two kinds of premise variables:

-Measurable premise variable (for example input, output)

-Unmeasurable premise variable (for example state)

We present in this paper the stability condition and design procedure of the augmented TS fuzzy system compound from TS fuzzy model and TS fuzzy observer. We show through this work the difference between the utilization of the same or different cases of premise variable (measurable or unmeasurable) for the TS fuzzy model construction and for the TS fuzzy observer design.

We conclude that we obtain the same stability conditions if we use premise variables with same nature for TS fuzzy model and TS fuzzy observer.

We make an indication and correction of a fault made in paper [1] [8] which deal with the unmeasurable premise variable case

We summarize our contribution in this paper as:

- We present a new classification about measurable and unmeasurable premise variable for augmented system design (TS fuzzy model and fuzzy observer).

- We prove that the use of the same nature of the premise variable (measurable or unmeasurable) for TS fuzzy model and fuzzy observer has the same stability conditions

- We make a correction for the theorem proposed by TANAKA and al. [1], in the design of fuzzy observer with unmeasurable premise variable case.

\section{References}

[1] K. Tanaka, T. Ikeda and H. O. Wang, "Fuzzy Regulators and Fuzzy Observers: Relaxed Stability Conditions and LMI-Based Designs", IEEE Tra. On Fuzzy Systems, vol. 6, no. 2, (1998), pp.250-265.

[2] X. J. Ma, Z. Q. Sun and Y. Y. He, "Analysis and design of fuzzy controller and fuzzy observer", IEEE Trans. Fuzzy Sys., vol. 6, no. 1, (1998), pp. 41-51.

[3] A. Akhenak, "Conception des observateurs non lineaires par approche multi-modele : application au diagnostic", PHD thesis, Ecole doctorale IAEM Lorraine, France, (2004).

[4] R.J Patton, J. Chen and C. J. Lopez-Toribio, "design a fault diagnosis scheme using TS-fuzzy observer", Proc. Of the 37th IEEE CDC Tampa, Florida USA, (1998), pp. 84-89

[5] D. Ichalal, B. Marx, J. Ragot and D. Maquin, "State estimation of Takagi-Sugeno systems with unmeasurable premise variables", IET Control TheoryAppl., vol. 4, no. 5, (2010), pp. 897-908.

[6] T. Youssef, M. Chadli, H.R. Karimi and M. Zelmat, "Design of unknown inputs proportional integral observers for TS fuzzy models", Neuro-computing, vol. 123, (2014), pp. 156-165.

[7] P. Bergsten and R. PALM, "Thau-Luenberger observers for TS fuzzy systems". In 9th IEEE Int. Conf. on Fuzzy Systems, San Antonio, Texas, USA, (2000).

[8] K. Tanaka, Hua O. Wang, "Fuzzy Control Systems Design and Analysis; A Linear Matrix Inequality Approach", John Wiley Sons, Inc. New York (2001), ISBN: 0-471-22459-6.

[9] D. Ichalal. "Estimation et diagnostic de systèmes non linéaires d'écrits par un modèle de TakagiSugeno ", doctorat thesis, Nancy university INPL, France, (2009). 
[10] P. Bergsten, R. Palm and D. Driankov, “Observers for Takagi-Sugeno Fuzzy Systems”, IEEE TRA. On system, Man, AND Cybernetics -PART B: CYBERNETICS, vol. 32, no. 1, (2002), pp. 114-121.

[11] H.O. Wang, K. Tanaka and M. F. Griffin, "An approach to Fuzzy Control of nonlinear systems: Stability and Design Issues", IEEE Trans. on Fuzzy Systems, vol. 4, no. 1, (1996), pp. 14-23.

[12] M. C. M. Teixeira and S. H. Zak, "Stabilizing Controller Design for Uncertain Nonlinear Systems Using Fuzzy Models", IEEE Transaction on Fuzzy Systems, vol. 7, no. 2, (1999), pp. 133-142.

[13] G. Feng, "A Survey on Analysis and Design of Model-Based Fuzzy Control Systems", IEEE Trans. Fuzzy Systems, vol. 14, no. 5, (2006), pp. 676-697.

[14] H. Ballesteros-Moncada, E. J. Herrera-López and J. Anzurez-Marín, "Fuzzy model-based observers for fault detection in CSTR", ISA Transactions, vol. 59, (2015), pp.325-333.

[15] K. Tanaka and M. Sano, "On the concept of fuzzy regulators and fuzzy observers", in Proc. 3rd IEEE Int. Conf. Fuzzy Syst., Orlando, FL, vol. 2, (1994), pp. 767-772.

[16] H. Moodi and M. Farrokhi, "On observer-based controller design for Sugeno systems with unmeasurable premise variables”, ISA Transaction, vol. 53, no. 2, (2014), pp. 305-316,

[17] C. Liu, H.K. Lam, S.-H. Tsai and C.-S. Chen, "Control of nonlinear systems by fuzzy observercontroller with unmeasurable premise variable", Int. Conference on advanced robotics and intelligent systems (ARIS), Taipei, Taiwan, (2015).

\begin{abstract}
Authors
Mohamed Hazerchi, he received the B. Eng. and Master degree in Control Engineering from "Ecole Nationale Polytechnique (ENP)" of Algiers, in 2012. He is currently a $\mathrm{PhD}$ candidate at the "Laboratoire de Commande des Processus (LCP)", Department of Control, ENP of Algiers, Algeria. His research interests include fuzzy systems modeling, control and fuzzy fault detection and isolation, optimization problems.
\end{abstract}

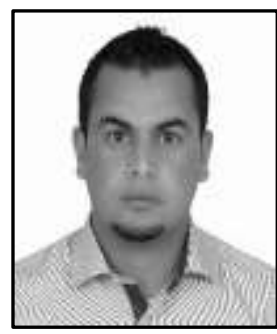

E-mail: hazerchimed@yahoo.com.

Rachid Illoul, he is an associate professor at "Ecole Nationale Polytechnique (ENP)" of Algiers, he is a member in the "laboratoire de commande des processus (LCP)", Department of Control, ENP of Algiers, Algeria. His research interests include fuzzy systems, nonlinear systems, neural network and optimization problems.

E-mail: rachid.illoul@enp.edu.dz. 
International Journal of Control and Automation

Vol. 11, No. 2 (2018) 